\title{
PERCEPCIÓN DEL ESTADO DE SALUD Y ESTILO DE VIDA EN LÍDERES RELIGIOSOS DE LIMA, PERÚ
}

\author{
Daniel Richard Pérez ${ }^{1}$
}

\begin{abstract}
Resumen
Objetivo. Determinar la relación entre la percepción del Estado de Salud y el Estilo de Vida en una población de líderes adventistas. Método. Tipo de estudio descriptivo-correlacional con diseño no experimental de corte transversal, llevado a cabo con 192 líderes religiosos. Los instrumentos aplicados fueron: Encuesta General de Salud de MOS (SF-12) y Cuestionario (modificado) de Perfil de Estilo de Vida (PEPS-I) utilizados por su alto grado de confiabilidad y validez (Alfa ce Cronbach) de ,818 y ,923 respectivamente. El análisis factorial exploratorio (KMO) fue de ,828 y ,851. Resultados: Se encontró que los líderes religiosos del sexo femenino perciben mejor su Estado de Salud (40,0\%) y manifiestan un mejor Estilo de Vida (31,4\%): Las variables sexo, edad, estado civil, región de procedencia, título alcanzado y área de desempeño no se relacionan con el Estilo de Vida ni con la percepción del Estado de Salud; únicamente tienen relación significativa con el Estilo de Vida las variables institución donde labora (0.031). Existe diferencias entre la actividad física, autocuidado, confianza en Dios y relaciones interpersonales respecto a la institución donde labora, siendo los líderes de la MICOP, APCS Y UPeU los que realizan mayor actividad física; APCE y UPeU, mejor autocuidado; APCE, UPeU y APCS, mayor confianza en Dios. Solo la UPeU se destaca en relaciones interpersonales. Existe diferencia $(\mathrm{p}=0,089<0,10)$ entre la institución donde labora y el rol emocional, siendo los obreros de la APCE, APCS Y UPEU los que manifiestan un mejor rol emocional. Conclusión: El Estilo de Vida solo se relaciona con la institución donde laboran los líderes religiosos. También existe relación entre el rol emocional y la institución donde labora. Por último, los líderes de la $\mathrm{UPeU}$ manifiestan tener mejores relaciones interpersonales.
\end{abstract}

Palabras clave: Estado de salud, estilo de vida, líderes religiosos

${ }^{1}$ Doctor en Teología y Salud Pública. Profesor de Teología, Investigación y Salud Pública en la Facultad de Teología de la Universidad Peruana Unión; Lima, Perú. Email: danielr@upeu.edu.pe 


\title{
PERCEPTION OF HEALTH STATUS AND LIFESTYLE IN RELIGIOUS LEADERS OF LIMA, PERU
}

\begin{abstract}
Aim: To determine the relationship between the perception of Health and Lifestyle in a population of Adventist leaders. Methodology: It's a descriptive-correlational study with no experimental cross-sectional design, conducted with 192 religious leaders. The instruments used were: General Health Survey (MOS SF-12) and Questionnaire (modified) Profile Lifestyle (PEPS-I) used for its high degree of reliability and validity (Alfa ce Cronbach) of ,818 and ,923 respectively. The exploratory factor analysis (KMO) FUW of ,828 and ,851. Results: It was found that female religious leaders better perceive their health status $(40.0 \%)$ and manifest a better lifestyle (31.4\%): The variables sex, age, marital status, region of origin, title reached and performance area are not related to the Lifestyle or the perception of the state of health; only they have significant relationship with variables Lifestyle institution where he works (0.031). There are differences between physical activity, self-care, trust in God and interpersonal relationships regarding the institution where he works, being leaders MICOP, APCS and UPeU those doing more physical activity; APCE and UPeU, better self-care; APCE, UPeU and APCS, greater trust in God. UPeU only excels in interpersonal relationships. There is a difference $(\mathrm{p}=0.089<0.10)$ between the institution where he works and emotional role, being the workers of the APCE, APCS and UPeU those who show better emotional role. Conclusion: Lifestyle only relates to the institution where religious leaders working. There is also a relationship between emotional role and the institution where he works. Finally, UPeU leaders say they have better interpersonal relationships.
\end{abstract}

Keywords: Health Status, Lifestyle, Religious leaders 


\section{Introducción}

Kenneth Cooper, creador del famoso Instituto Aeróbico de Dallas, afirmó: "Ahora ya no morimos de una enfermedad, sino que morimos por la forma en que vivimos". ${ }^{1}$ Esta declaración corrobora los numerosos informes de la OMS y la OPS al manifestar que los problemas globales de salud que predominan en la actualidad, y que son las primeras causas de morbimortalidad, son el resultado de las conductas de salud que comprometen la salud de la población. ${ }^{2}$

El fenómeno de la globalización y los cambios demográficos y socioeconómicos ocurridos desde fines del siglo XX y comienzos del XXI, han modificado notoriamente el comportamiento de las personas, dando lugar a nuevos patrones epidemiológicos en el que disminuyen las enfermedades infecto-contagiosas y se incrementan las enfermedades crónicas no trasmisibles (ECNT). ${ }^{3}$

Este hecho motiva la realización del presente trabajo cuyo obje10 tivo es encontrar la relación de la percepción del Estado de Salud con el Estilo de Vida en una población de líderes religiosos adventistas.

Estado de Salud, se define como la "percepción subjetiva que tiene la persona sobre su estado de salud general". ${ }^{4}$ Se distinguen 5 niveles: Mala, Regular, Buena, Muy buena y Excelente. Los epidemiólogos tradicionales conceptualizan Estado de Salud como "presencia de enfermedad" o "ausencia de enfermedad", el cual es una definición muy simple debido a que los criterios de diagnóstico cambian muy rápido a medida que au-

${ }^{1}$ Daniel Richard, Educción para la salud (Lima: Editoral Imprenta Unión, 2013).

${ }^{2}$ Nola, J. Pender, El modelo de la promoción de la salud (Connecticut: Appleton \& Lange, 1996).

${ }^{3}$ Organización Panamerica de la Salud, "Calidad de vida y Estilo de vida”, Rev Panam Salud Publica 3/1 (1998): 20-35.

${ }^{4}$ Instituto Nacional de Estadística (INE). Encuesta Europea de Salud en España. Ministerio de Sanidad Política social e Igualdad, 2009. 
mentan los conocimientos y mejoran las técnicas. ${ }^{5}$ Si bien en la mayoría de los países la esperanza de vida se ha incrementado por la baja mortalidad infantil; sin embargo, esta situación no necesariamente indica que el Estado de Salud de las personas haya mejorado. ${ }^{6}$

Sobre Estilo de Vida, existen diversas definiciones debido a las distintas disciplinas que utilizan el término; aun así, la OMS la define como: "Forma general de vida basada en la interacción entre las condiciones de vida en un sentido amplio y los patrones individuales de conducta determinados por factores socioculturales y características personales". ${ }^{7}$ Otra definición es la enunciada por Nancy Milio quien la conceptualiza como "el conjunto de patrones de conducta, escogidas a partir de las alternativas que están disponibles a las personas, de acuerdo a sus circunstancias socioeconómicas y a las facilidades con las cuales son capaces de escoger entre las distintas opciones". ${ }^{8}$ Esta definición resalta elementos importantes como son: la forma como el individuo interactúa con su medioambiente, su vida diaria, sus valores, actitudes, opiniones, patrones de comportamiento e intereses; es decir, el modus vivendi de la persona.

Finalmente, los resultados del estudio permiten incrementar el conocimiento sobre la percepción del Estado de Salud y la Calidad de Vida en una muestra sui géneris como son los líderes religiosos, que a su vez servirá como línea de base para la implementación de estrategias orientadas a la promoción de la salud con el fin de mejorar la calidad de vida de las personas y la sociedad en general.

${ }^{5}$ R. J. Prineas et al., The Minnesota Codo Manual or Electrocardiographic Findings: Standards and Procedures for Measurement and Classification (Stoneham, MA: Butterworth, 1982).

${ }^{6}$ A. L. González-Celis, "Efectos de intervención de un programa de promoción a la salud sobre la calidad de vida en ancianos" (Tesis de doctorado, México: Facultad de Psicología, UNAM, 2002).

${ }^{7}$ [I. Kickbusch], World Health Organization, "Life-Styles and Health", Social Science \& Medicine 22/2 (1986) 117-124.

${ }^{8}$ N. Milio, Promoting Health through Public Policy (Philadelphia: FA Davis. 1981). 


\section{Materiales y método}

La investigación es de tipo descriptiva correlacional. Busca relacionar los constructos Percepción del Estado de Salud y Estilo de Vida en una población de 192 líderes de la Iglesia Adventista del Séptimo Día (IASD) de tres campos misioneros y una institución educativa: Asociación Peruana Central Sur (APCS), Asociación Peruana Central Este (APCE), Misión Centro Oeste del Perú (MICOP) y Universidad Peruana Unión (UPeU) sede Lima. El estudio tiene un diseño no experimental y de corte transversal.

Con el fin de alcanzar los objetivos planteados se aplicaron los cuestionarios mencionados que en su parte inicial incluyó la siguiente información demográfica: sexo, edad, estado civil, máximo título alcanzado, región de procedencia, número de hijos, peso, talla, institución donde labora, y años de servicio en la IASD.

La información fue recopilada mediante dos instrumentos: (1) Cues-

12 tionario sobre Estado de Salud SF-12, y (2) Cuestionario (modificado) de Perfil de Estilo de Vida (PEPS-I) de Pender, 1996; complementado con el Cuestionario de Prácticas y Creencias sobre Estilos de Vida de Arrivillaga y Salazar.?

El Cuestionario sobre Estado de Salud (SF-12), es la versión reducida del SF-36, originalmente denominado Medical Outcomes Survey (MOS) Short-Form General Health Survey (SF-12).

El SF-12 fue adaptado al español por Alonso, Prieto y Antó, ${ }^{10}$ e incluye 12 preguntas que evalúa la percepción que la persona tiene sobre su estado de salud en ocho aspectos: Función física ( 2 ítems), Rol físico (2 ítems), Dolor corporal (1 ítem), Salud general (1 ítem),

${ }^{9}$ M. Arrivillaga y I. Salazar, "Creencias relacionadas con el estilo de vida de jóvenes latinoamericanos", Rv. Internacional de Psicología Clinica y de la Salud 13/1 (2005): 19-36.

${ }^{10} \mathrm{~J}$. Alonso, L. Prieto y J. M. Antó, La versión española del SF-36 Health Survey (Cuestionario de Salud SF-36): Un instrumento para la medida de los resultados clinicos (Barcelona: Med Clin, 1995), 104:771-6. 
Vitalidad (1 ítem), Función social (1 ítem), Rol emocional (2 ítems), y Salud mental (2 ítems). Las alternativas de respuestas del SF-12 están diseñadas en un formato tipo LIKERT en escala ordinal y con diversas valoraciones de respuestas tales como: Mala (1), Regular (2), Buena (3), Muy buena (4), Excelente (5); Sí, me limita mucho (1), Sí, me limita un poco (2) y No, no me limita nada (3); Sí (1) y No (2); Nada (1), Un poco (2), Regular (3), Bastante (4), Mucho (5); Nunca (1), Solo alguna vez (2), Algunas veces (3), Muchas veces (4), Casi siempre (5), y Siempre (6). Para evaluar la fiabilidad y validez del instrumento se aplicó a 223 líderes de otros campos e instituciones adventistas de Perú entre los 22 y 68 ańos, la consistencia de sus dimensiones se muestra con un alfa de Cronbach global de 0.81. La evaluación de la estructura factorial y de constructo de los instrumentos se realizaron con el análisis factorial exploratorio, observando que en los tres instrumentos el coeficiente KMO toma valores superiores a 0.80 , indicando que los ítems pueden reducirse a dimensiones, además la varianza total explicada al reducir sus dimensiones explican más del $45 \%$ y la mayoría de los ítems ingresan en sus respectivas dimensiones.

El Cuestionario sobre Estilo de Vida consta de 77 preguntas distribuidas en siete dimensiones, que evalúa el nivel de prácticas de los principales hábitos de estilo de vida en los siguientes aspectos: Actividad física y deporte (5 ítems), Recreación y descanso (15 ítems), Autocuidado (10 ítems), Hábitos alimenticios (20 ítems), Confianza en Dios (5 ítems), Relaciones interpersonales (9 ítems) y Bienestar psicológico (13 ítems). Las alternativas de respuestas del cuestionario de estilo de vida fueron diseńadas en un formato tipo LIKERT en escala ordinal y con 4 valoraciones de respuestas: Nunca (1), Algunas veces (2), A menudo (3), y Siempre (4). Para evaluar la fiabilidad y validez del instrumento se aplicó a 223 líderes de instituciones adventistas de otros campos de Perú entre los 22 y 68 años, la consistencia de sus dimensiones se muestra con un alfa de Cronbach global de 0.92 que 
es valorado como alto o consistente; se hizo lo mismo con los siete componentes del instrumento: $0.803,0.696,0.883,0.767,0.652$, 0.799 , y 0.870 respectivamente.

\section{Resultados}

\section{Características sociodemográficas}

La tabla 1 describe las principales características sociodemográficas de la población estudiada.

Tabla 1. Entorno demográfico 1

\begin{tabular}{|c|c|c|}
\hline Características sociodemográficas & $\mathrm{n}$ & $\%$ \\
\hline \multicolumn{3}{|l|}{ 1. Sexo } \\
\hline Hombre & 157 & $81.8 \%$ \\
\hline Mujer & 35 & $18.2 \%$ \\
\hline \multicolumn{3}{|l|}{ 2. Estado civil } \\
\hline Soltero(a) & 13 & $6.8 \%$ \\
\hline Casado(a) & 176 & $92.1 \%$ \\
\hline Viudo(a) & 2 & $1.0 \%$ \\
\hline Divorciado(a) & 0 & $0.0 \%$ \\
\hline \multicolumn{3}{|l|}{ 3. Región de procedencia } \\
\hline Costa & 82 & $43.4 \%$ \\
\hline Sierra & 77 & $40.7 \%$ \\
\hline Selva & 30 & $15.9 \%$ \\
\hline \multicolumn{3}{|c|}{ 4. Máximo título/grado alcanzado } \\
\hline Título técnico & 14 & $7.4 \%$ \\
\hline Bachiller & 38 & $20.0 \%$ \\
\hline Título universitario & 75 & $39.5 \%$ \\
\hline
\end{tabular}




\begin{tabular}{|l|r|r|}
\hline Maestría & 51 & $26.8 \%$ \\
\hline Doctorado & 12 & $6.3 \%$ \\
\hline 5. Institución donde labora & \multicolumn{2}{|c|}{} \\
\hline APCE & 62 & $32.3 \%$ \\
\hline MICOP & 32 & $16.7 \%$ \\
\hline UPeU & 42 & $21.9 \%$ \\
\hline APCS & 56 & $29.2 \%$ \\
\hline 6. Área de desempeño profesional actual & \multicolumn{3}{|c|}{} \\
\hline Pastoral & 117 & $62.2 \%$ \\
\hline Administrativa & 17 & $9.0 \%$ \\
\hline Educativa & 35 & $18.6 \%$ \\
\hline Salud & 2 & $1.1 \%$ \\
\hline Otro & 17 & $9.0 \%$ \\
\hline
\end{tabular}

$\mathrm{n}=192$

La tabla 2, muestra la media, desviación estándar, mínimo y máximo de: edad, número de hijos, peso, talla, y ańos de servicio en la IASD.

Tabla 2. Entorno demográfico 2

\begin{tabular}{|l|r|r|r|r|}
\hline & \multicolumn{1}{|c|}{ Media } & \multicolumn{1}{|c|}{$\begin{array}{l}\text { Desviación } \\
\text { estándar }\end{array}$} & \multicolumn{1}{c|}{ Mínimo } & \multicolumn{1}{c|}{ Máximo } \\
\hline Edad & 44 & 12 & 31 & 68 \\
\hline $\begin{array}{l}\text { Número de } \\
\text { hijos }\end{array}$ & 2 & 1 & 0 & 5 \\
\hline Peso & 71 & 11 & 49 & 108 \\
\hline Talla & 1,66 & 0,07 & 1,50 & 1,87 \\
\hline $\begin{array}{l}\text { Años de servicio } \\
\text { en la IASD }\end{array}$ & 16,33 & 1,75 & 1 & 43 \\
\hline
\end{tabular}

$\mathrm{n}=192$ 


\section{Distribución de las características demográficas respecto al Estilo de Vida y el Estado de Salud}

En la tabla 3 se observa que la mayoría de damas manifiestan tener un mejor Estado de Salud (40,0\%) y un mejor Estilo de Vida (31,4\%) en relación a los varones.

Tabla 3. Estilo de vida y estado de salud respecto al sexo

\begin{tabular}{|l|r|r|r|r|r|c|}
\hline \multirow{2}{*}{} & \multicolumn{3}{|c|}{ Hombre \% del n } & \multicolumn{3}{c|}{ Mujer \% del n } \\
\cline { 2 - 7 } & Alto & \multicolumn{1}{c|}{ Medio } & \multicolumn{1}{c|}{ Bajo } & \multicolumn{1}{c|}{ Alto } & \multicolumn{1}{c|}{ Medio } & \multicolumn{1}{c|}{ Bajo } \\
\hline $\begin{array}{l}\text { Estado } \\
\text { de Salud }\end{array}$ & $36,9 \%$ & $44,6 \%$ & $18,5 \%$ & $40,0 \%$ & $34,3 \%$ & $25,7 \%$ \\
\hline $\begin{array}{l}\text { Estilo } \\
\text { de Vida }\end{array}$ & $26,8 \%$ & $39,5 \%$ & $33.8 \%$ & $31,4 \%$ & $48,6 \%$ & $20,0 \%$ \\
\hline
\end{tabular}

$\mathrm{n}=192$

Según la tabla 4, las personas viudas $(100 \%)$ y solteras $(61,5 \%)$ perciben mejor su Estado de Salud; casi lo mismo ocurre en cuanto al Estilo de Vida.

Tabla 4. Estilo de vida y estado de salud respecto al estado civil

\begin{tabular}{|c|c|c|c|c|c|}
\hline \multirow{2}{*}{\multicolumn{2}{|c|}{ Soltero $(a) \%$ del n }} & \multicolumn{4}{|c|}{ Estado civil } \\
\hline & & \multirow{2}{*}{$\begin{array}{l}\text { Soltero(a) } \\
\% \text { del } \mathrm{n} \\
\end{array}$} & \multirow{2}{*}{$\begin{array}{l}\text { Casado(a) } \\
\% \text { del n }\end{array}$} & \multirow{2}{*}{$\begin{array}{l}\text { Viudo(a) } \\
\% \text { del } \mathrm{n} \\
\end{array}$} & \multirow{2}{*}{$\begin{array}{c}\begin{array}{c}\text { Divorcia- } \\
\text { do(a) }\end{array} \\
\% \text { del } \mathrm{n} \\
\end{array}$} \\
\hline & & & & & \\
\hline \multirow{3}{*}{$\begin{array}{l}\text { Estilo } \\
\text { de Vida }\end{array}$} & Bajo & $38,5 \%$ & $31,3 \%$ & $0,0 \%$ & $0,0 \%$ \\
\hline & Medio & $30,8 \%$ & $41,5 \%$ & $50,0 \%$ & $0,0 \%$ \\
\hline & Alto & $30,8 \%$ & $27,3 \%$ & $50,0 \%$ & $0,0 \%$ \\
\hline
\end{tabular}




\begin{tabular}{|l|l|r|r|r|r|}
\hline \multirow{3}{*}{$\begin{array}{l}\text { Estado } \\
\text { de Salud }\end{array}$} & Bajo & $0,0 \%$ & $21,6 \%$ & $0,0 \%$ & $0,0 \%$ \\
\cline { 2 - 6 } & Medio & $38,5 \%$ & $43,2 \%$ & $0,0 \%$ & $0,0 \%$ \\
\cline { 2 - 6 } & Alto & $61,5 \%$ & $35,2 \%$ & $100,0 \%$ & $0,0 \%$ \\
\hline
\end{tabular}

$\mathrm{n}=192$

La tabla 5 presenta un Estado de Salud más alto para los de la costa $(41,5 \%)$ y la selva $(36,7 \%)$, repitiéndose en mismo fenómeno en relación al Estilo de Vida.

Tabla 5. Estilo de vida y estado de salud respecto a la región de procedencia

\begin{tabular}{|c|c|c|c|c|}
\hline & \multicolumn{3}{|c|}{ Región de procedencia } \\
\hline & & Costa & Sierra & Selva \\
\hline & & $\%$ del $n$ & $\%$ del $n$ & $\%$ del $n$ \\
\hline \multirow[t]{3}{*}{ Estilo de Vida } & Bajo & $31,7 \%$ & $31,2 \%$ & $30,0 \%$ \\
\hline & Medio & $37,8 \%$ & $44,2 \%$ & $40,0 \%$ \\
\hline & Alto & $30,5 \%$ & $24,7 \%$ & $30,0 \%$ \\
\hline \multirow[t]{3}{*}{ Estado de Salud } & Bajo & $19,5 \%$ & $20,8 \%$ & $13,3 \%$ \\
\hline & Medio & $39,0 \%$ & $44,2 \%$ & $50,0 \%$ \\
\hline & Alto & $41,5 \%$ & $35,1 \%$ & $36,7 \%$ \\
\hline
\end{tabular}

$\mathrm{n}=192$

En la tabla 6 se percibe que el Estado de Salud y el Estilo de vida están en sus niveles más altos en quienes al alcanzado el grado doctoral $(58,3 \%-35,3 \%)$ y la maestría $(41,7 \%$ - 39,2\%). Ello es corroborado por numerosos estudios en los se muestra que a mayor nivel de estudios mejor calidad de vida, ya que el nivel de estudio es un requisito para alcanzar un mejor empleo y mejores ganancias. 
Daniel Richard Pérez

Tabla 6. Estilo de vida y estado de salud respecto al máximo título alcanzado

\begin{tabular}{|l|l|r|r|r|r|r|}
\hline \multirow{2}{*}{} & \multicolumn{5}{|c|}{ Máximo título/grado alcanzado } \\
\cline { 3 - 7 } & $\begin{array}{c}\text { Título } \\
\text { técnico }\end{array}$ & Bachiller & $\begin{array}{c}\text { Título uni- } \\
\text { versitario }\end{array}$ & Maestría & Doctorado \\
\cline { 3 - 7 } & $\%$ del n & $\%$ del n & $\%$ del n & $\%$ del n & \\
\hline \multirow{2}{*}{$\begin{array}{l}\text { Estilo } \\
\text { de Vida }\end{array}$} & Bajo & $50,0 \%$ & $44,7 \%$ & $29,3 \%$ & $21,6 \%$ & $16,7 \%$ \\
\cline { 2 - 7 } & Medio & $42,9 \%$ & $36,8 \%$ & $41,3 \%$ & $43,1 \%$ & $41,7 \%$ \\
\cline { 2 - 7 } & Alto & $7,1 \%$ & $18,4 \%$ & $29,3 \%$ & $35,3 \%$ & $41,7 \%$ \\
\hline \multirow{2}{*}{$\begin{array}{l}\text { Estado } \\
\text { de Salud }\end{array}$} & Bajo & $42,9 \%$ & $18,4 \%$ & $12,0 \%$ & $23,5 \%$ & $16,7 \%$ \\
\cline { 2 - 7 } & Medio & $28,6 \%$ & $47,4 \%$ & $50,7 \%$ & $37,3 \%$ & $25,0 \%$ \\
\cline { 2 - 7 } & Alto & $28,6 \%$ & $34,2 \%$ & $37,3 \%$ & $39,2 \%$ & $58,3 \%$ \\
\hline
\end{tabular}

$\mathrm{n}=192$

Según la tabla 7, la mayoría de los líderes de la MICOP $(40,6 \%)$ y 18 la UPeU $(38,1 \%)$ manifiestan un mejor Estado de Salud; en cuanto al Estilo de Vida, se destacan la UPeU (40,5\%) seguido de la APCS $(33,9 \%)$.

Tabla 7. Estilo de vida y estado de salud respecto a la institución donde labora

\begin{tabular}{|c|c|c|c|c|c|}
\hline & \multicolumn{4}{|c|}{ Institución donde labora } \\
\hline & & APCS & APCE & MICOP & UPEU \\
\hline & & $\%$ del $n$ & $\%$ del $n$ & $\%$ del $\mathrm{n}$ & $\%$ del $n$ \\
\hline \multirow{3}{*}{$\begin{array}{l}\text { Estilo } \\
\text { de Vida }\end{array}$} & Bajo & $37,5 \%$ & $27,4 \%$ & $37,5 \%$ & $23,8 \%$ \\
\hline & Medio & $28,6 \%$ & $54,8 \%$ & $43,8 \%$ & $35,7 \%$ \\
\hline & Alto & $33,9 \%$ & $17,7 \%$ & $18,8 \%$ & $40,5 \%$ \\
\hline \multirow{3}{*}{$\begin{array}{l}\text { Estado } \\
\text { de salud }\end{array}$} & Bajo & $23,2 \%$ & $19,4 \%$ & $12,5 \%$ & $21,4 \%$ \\
\hline & Medio & $41,1 \%$ & $43,5 \%$ & $46,9 \%$ & $40,5 \%$ \\
\hline & Alto & $35,7 \%$ & $37,1 \%$ & $40,6 \%$ & $38,1 \%$ \\
\hline
\end{tabular}

$\mathrm{n}=192$ 
La tabla 8 muestra que el mejor Estado de Salud es manifestado por los líderes del área de salud (50\%) seguido del área administrativa (41.2\%); en cuanto a Calidad de Vida, mejor están los del área de salud (50\%) seguido del área educativa $(37,1 \%)$.

Tabla 8. Estilo de vida y estado de salud respecto

al área de desempeño profesional

\begin{tabular}{|c|c|c|c|c|c|c|}
\hline & \multicolumn{5}{|c|}{ Área de desempeńo profesional actual } \\
\hline & & Pastoral & Administrativa & Educativa & Salud & Otro \\
\hline & & $\%$ del $n$ & $\%$ del $n$ & $\%$ del $n$ & $\%$ del $n$ & $\%$ del $n$ \\
\hline \multirow{3}{*}{$\begin{array}{l}\text { Estilo } \\
\text { de Vida }\end{array}$} & Bajo & $36,8 \%$ & $41,2 \%$ & $14,3 \%$ & $0,0 \%$ & $23,5 \%$ \\
\hline & Medio & $35,0 \%$ & $41,2 \%$ & $48,6 \%$ & $50,0 \%$ & $64,7 \%$ \\
\hline & Alto & $28,2 \%$ & $17,6 \%$ & $37,1 \%$ & $50,0 \%$ & $11,8 \%$ \\
\hline \multirow{3}{*}{$\begin{array}{l}\text { Estado } \\
\text { de salud }\end{array}$} & Bajo & $21,4 \%$ & $17,6 \%$ & $20,0 \%$ & $0,0 \%$ & $11,8 \%$ \\
\hline & Medio & $40,2 \%$ & $41,2 \%$ & $45,7 \%$ & $50,0 \%$ & $58,8 \%$ \\
\hline & Alto & $38,5 \%$ & $41,2 \%$ & $34,3 \%$ & $50,0 \%$ & $29,4 \%$ \\
\hline
\end{tabular}

$\mathrm{n}=192$

\section{Pruebas de relación entre las características demográficas, el Estilo de Vida y el Estado de Salud}

La tabla 9 muestra que existe relación significativa entre el Estilo de Vida y la institución donde labora $(0,031)$; también existe relación aunque no tan significativa, entre el estado civil y el Estado de Salud $(0,076)$. 
Tabla 9. Prueba Chi-cuadrada de la relación entre características demográficas, Estado de Salud y Estilo de Vida

\begin{tabular}{|l|r|r|r|r|r|r|}
\hline & \multicolumn{3}{|c|}{ Estilo de vida } & \multicolumn{3}{c|}{ Estado de salud } \\
\hline $\begin{array}{l}\text { Características } \\
\text { demográficas }\end{array}$ & $\begin{array}{c}\text { Chicua- } \\
\text { drado }\end{array}$ & gl & \multicolumn{1}{c|}{ Sig } & $\begin{array}{c}\text { Chicua- } \\
\text { drado }\end{array}$ & gl & Sig \\
\hline Sexo & 2,534 & 2 & 0,282 & 1,541 & 2 & 0,463 \\
\hline Estado civil & 1,631 & 4 & $0,803^{\text {a,b }}$ & 8,464 & 4 & $0,076^{\mathrm{a}, \mathrm{b}}$ \\
\hline Región de procedencia & 0,946 & 4 & 0,918 & 1,759 & 4 & 0,780 \\
\hline $\begin{array}{l}\text { Máximo } \\
\text { título/grado } \\
\text { alcanzado }\end{array}$ & 11,747 & 8 & $0,163^{\mathrm{a}}$ & 11,703 & 8 & $0,165^{\mathrm{a}}$ \\
\hline $\begin{array}{l}\text { Institución donde } \\
\text { labora }\end{array}$ & 13,892 & 6 & $0,031^{*, \mathrm{~b}}$ & 1,616 & 6 & $0,951^{\mathrm{b}}$ \\
\hline $\begin{array}{l}\text { Área de desempeño } \\
\text { profesional } \\
\text { actual }\end{array}$ & 13,372 & 8 & $0,100^{\mathrm{a}, \mathrm{b}}$ & 3,009 & 8 & $0,934^{\mathrm{a}, \mathrm{b}}$ \\
\hline
\end{tabular}

Pruebas de relación entre "institución donde labora" y las dimensiones del Estilo de Vida (actividad física, autocuidado, confianza en Dios y relaciones interpersonales), y del Estado de Salud (rol emocional)

Según las tablas 10,11 y 12 existe diferencia $(\mathrm{p}=0,054<0,10)$ entre la institución en la cual laboran los líderes religiosos y el Estilo de Vida que practican. Se destaca la UPeU por tener un mejor Estilo de Vida respecto a las otras instituciones de Lima; asimismo, el análisis de varianza revela que existe diferencias entre las dimensiones del Estilo de Vida: actividad física, autocuidado, confianza en Dios, y relaciones interpersonales respecto a las instituciones donde laboran los participantes, siendo los obreros de la MICOP, APCS Y UPeU los que realizan mayor actividad fisica; APCE y UPeU, mejor autocuidado, APCE, UPeU y APCS, mayor confianza en Dios, y solo UPeU se destaca en relaciones interpersonales; 
además, existe diferencia $(\mathrm{p}=0,089<0,10)$ en la dimensión rol emocional de la Calidad de Vida, siendo los obreros de la APCE, APCS Y UPEU los que manifiestan un mejor rol emocional.

Tabla 10. Análisis de varianza entre las dimensiones del Estilo de Vida y el Estado de Salud, respecto a las instituciones donde labora

\begin{tabular}{|l|r|r|}
\hline & $\mathrm{F}(3,188)$ & Sig. \\
\hline Estilo de Vida & 2,588 & $\mathbf{0 5 4}$ \\
\hline Actividad fisica & 4,937 & $\mathbf{0 0 3}$ \\
\hline Recreación &, 769 &, 513 \\
\hline Autocuidado & 2,355 & $\mathbf{, 0 7 3}$ \\
\hline Hábitos alimenticios &, 885 &, 450 \\
\hline Confianza Dios & 2,836 &, $\mathbf{0 3 9}$ \\
\hline Relaciones interpersonales & 3,701 &, $\mathbf{0 1 3}$ \\
\hline Bienestar psicológico & 1,060 &, 368 \\
\hline Calidad de Vida &, 117 &, 950 \\
\hline Función fisica & 1,366 &, 255 \\
\hline Rol fisico &, 740 &, 530 \\
\hline Dolor corporal &, 633 &, 595 \\
\hline Salud general & 1,596 &, 194 \\
\hline Vitalidad &, 561 &, 641 \\
\hline Función social &, 329 &, 804 \\
\hline Rol emocional & 2,206 &, $\mathbf{0 8 9}$ \\
\hline Salud mental &, 674 &, 569 \\
\hline
\end{tabular}


Tabla 11. Prueba de comparaciones múltiples (Duncan) entre la institución donde labora y cuatro dimensiones del Estilo de Vida

\begin{tabular}{|c|c|c|c|c|c|}
\hline \multirow{2}{*}{ Institución donde labora } & \multirow{2}{*}{$\mathrm{n}$} & \multicolumn{2}{|c|}{ Subconjunto para alfa $=0.05$} & \multirow{2}{*}{\begin{tabular}{c|} 
Sig. \\
1
\end{tabular}} & \multirow[b]{2}{*}{2} \\
\hline & & 1 & 2 & & \\
\hline \multicolumn{2}{|l|}{ Estilo de vida } & & & ,308 &, 105 \\
\hline MICOP & 32 & 219,47 & & & \\
\hline APCE & 62 & 221,63 & & & \\
\hline APCS & 56 & 224,68 & 224,68 & & \\
\hline UPeU & 42 & & 232,45 & & \\
\hline \multicolumn{2}{|l|}{ Actividad fisica } & & & 254 &, 092 \\
\hline APCE & 62 & 9,81 & & & \\
\hline MICOP & 32 & 10,63 & 10,63 & & \\
\hline APCS & 56 & & 11,88 & & \\
\hline UPeU & 42 & & 11,90 & & \\
\hline \multicolumn{2}{|l|}{ Autocuidado } & & & ,608 &, 051 \\
\hline MICOP & 32 & 23,19 & & & \\
\hline APCS & 56 & 23,64 & & & \\
\hline APCE & 62 & 23,90 & 23,90 & & \\
\hline UPeU & 42 & & 26,45 & & \\
\hline Confianza Dios & & & & 1,000 & ,462 \\
\hline MICOP & 32 & 15,56 & & & \\
\hline APCE & 62 & & 16,58 & & \\
\hline UPeU & 42 & & 16,71 & & \\
\hline APCS & 56 & & 16,95 & & \\
\hline \multicolumn{2}{|l|}{ Relaciones interpersonales } & & &, 160 & 1,000 \\
\hline MICOP & 32 & 25,44 & & & \\
\hline APCE & 62 & 26,69 & & & \\
\hline APCS & 56 & 26,79 & & & \\
\hline UPeU & 42 & & 28,67 & & \\
\hline
\end{tabular}

$\mathrm{n}=192$ 
Tabla 12 - Prueba de comparaciones múltiples (Duncan) entre la institución donde labora y la dimensión Rol emocional del Estado de Salud

\begin{tabular}{|l|r|r|r|r|r|}
\hline \multirow{2}{*}{$\begin{array}{l}\text { Institución } \\
\text { donde labora }\end{array}$} & \multirow{2}{*}{$\mathrm{n}$} & \multicolumn{2}{|c|}{$\begin{array}{c}\text { Subconjunto para alfa } \\
0.05\end{array}$} & \multirow{2}{*}{ Sig. } & \\
\cline { 3 - 6 } & & 1 & 2 & 1 & 2 \\
\hline Rol emocional & & & & 1,000 &, 646 \\
\hline MICOP & 32 & 3,28 & & & \\
\hline APCE & 62 & & 3,60 & & \\
\hline APCS & 42 & & 3,64 & & \\
\hline UPeU & 55 & & 3,67 & & \\
\hline
\end{tabular}

$\mathrm{n}=192$

\section{Discusión}

Con esta investigación se busca verificar la relación entre el Estado de Salud y la Calidad de Vida en una población de líderes religiosos. La relación entre estos dos constructos ha sido corroborada por una serie de estudios. Sin embargo, nuestro el caso de esta investigación también se han encontrado relaciones entre algunas dimensiones del Estilo de Vida y el Estado de Salud con la variable demográfica institución donde laboran el líder religioso.

Las variables sexo, edad, estado civil, región de procedencia, título alcanzado y área de desempeño no se relacionan significativamente con el Estilo de Vida ni con la percepción del Estado de Salud; la única que si tiene relación significativa con el estilo de vida es la variable institución donde labora (0.031). Este dato difiere en parte con el estudio de Rebollo y Núnez, ${ }^{11}$ quienes manifiestan que si existe relación significativa del Estado de Salud El género y el nivel educativo.

${ }^{11}$ M. Rebollo y M. Núñez, La mirada de la otra: Calidad ce vida, género y educación (Sevilla, España: Universidad de Sevilla, 1998). 
Además, el hecho de que las dimensiones del estilo de vida muestran correlaciones positivas con la calidad de vida, es corroborado por el estudio de Jacoby, ${ }^{12}$ quien refiere que 30 minutos diarios de ejercicio físico de intensidad moderada cada día o durante casi todos los días de la semana proporcionan beneficios de salud importantes. Asimismo, la dimensión de recreación tiene una correlación positiva con la calidad de vida, ello debido a que el 55.6\% realiza algún tipo de actividad recreativa alta o media. También, los hábitos alimentarios, se correlacionan positivamente con la calidad de vida, esto podría justificarse debido a que los participantes reconocen la importancia que tiene los hábitos alimentarios en la salud. Sanabria-Ferrand ${ }^{13}$ señala que las personas que tienen mayores conocimientos de salud se dan cuenta que sus acciones tienen un impacto directo en la misma y se preocuparan por conocer lo que necesitan para mantenerse saludables. La dimensión confianza en Dios, también evidencia una correlación positiva por ser esta una actividad habitual de los participantes del estudio que en su mayoría son pastores y líderes espirituales. Por otro lado, las dimensiones relaciones interpersonales y bienestar psicológico, muestran una correlación positiva con la calidad de vida debido a que en el ambiente laboral de los sujetos de estudio existe permanente interacción.

Por otro lado, existe una correlación negativa de las dimensiones del nivel de estrés con la calidad de vida. Esto puede darse debido a que los participantes mayoritariamente tienen un estilo de vida saludable que se evidencia por la práctica permanente de buenos hábitos de salud, lo que hace que el estrés laboral y el cansancio emocional disminuya considerablemente o se evidencie en un porcentaje minoritario. Finalmente, se observa que la dimensión autocuidado de la variable estilo de vida no se

\footnotetext{
${ }^{12}$ Enrique Jacoby, Fiona Bull y Andrea Neiman, "Cambios acelerados del estilo de vida obligan a fomenar la actividad física como prioridad en la Región de las Américas", Rev. Panameriana de Salud Pública 14/4 (2003): 223-225.

${ }^{13}$ P. Sanabria-Ferrand, L. González y D. Urrego, "Estilos de vida saludables en profesionales colombianos de la salud. Estudio Exploratorio", Revista Médica 15/2 (2007): 207-217.
} 
relaciona con la calidad de vida, posiblemente porque es una variable que está íntimamente ligada con otras dimensiones del estilo de vida.

\section{Conclusión}

Por los resultados obtenidos se concluye que:

1) El sexo femenino presenta mejor estilo de vida, asimismo muestra que el sexo masculino posee más alto nivel de estrés laboral, además se presenta más alto nivel de calidad de vida en el sexo masculino

2) La mayoría de los participantes tienen un estilo de vida, nivel de estrés y calidad de vida en el nivel medio y bajo, independientemente de los factores demográficos.

3) Existe correlación negativa y significativa entre las dimensiones de estilo de vida y las dimensiones de nivel de estrés; asimismo, las dimensiones del estilo de vida muestran correlaciones positivas con la calidad de vida; al mismo tiempo se observa que la dimensión autocuidado del estilo de vida no se relaciona con la calidad de vida.

4) Finalmente, las distintas ecuaciones de regresión múltiple muestran que las dimensiones del estilo de vida (bienestar psicológico, hábitos alimenticios y relaciones interpersonales) se relacionan de manera positiva y estadísticamente significativa con la calidad de vida; en tanto que las dimensiones, cansancio físico, autocuidado y realización personal se relacionan de manera negativa pero estadísticamente significativa.

Recibido: 11/10/2015

Aceptado: 15/01/2016 